\title{
Textbook Outcomes Following Liver Resection for Cancer: A New Standard for Quality Benchmarking and Patient Decision Making
}

\author{
Jason Denbo, MD, CMQ, and Daniel A. Anaya, MD, FACS \\ Section of Hepatobiliary Tumors, Department of Gastrointestinal Oncology, H. Lee Moffitt Cancer Center and Research \\ Institute, Tampa, FL
}

In this issue of Annals of Surgical Oncology, Tsilimigras et al. assessed textbook outcomes (TO) following liver resection for primary liver tumors (intrahepatic cholangiocarcinoma [ICC]; hepatocellular carcinoma [HCC]) from major hepatobiliary centers across North America, Europe, Asia, and Australia, over a 12-year period. A TO was achieved when patients experienced (1) negativemargin liver resection; (2) no serious postoperative complications (Clavien-Dindo $\geq 3$ ); (3) no 30-day readmission; (4) no 30-day mortality; and (5) no prolonged length of stay (LOS). Sixty-two percent of patients achieved a TO, and TO was independently associated with a decreased risk of death for ICC and HCC. ${ }^{1}$

The concept of using a 'textbook outcome' following surgery to measure the quality or success of an operation is relatively new, although it has already gained strength as an important quality indicator for different surgical procedures. $^{2},{ }^{3}$ Essentially, a TO is a composite measure that was created by aggregating various perioperative outcomes believed to contribute to optimal results following an operation. The findings presented by Tsilimigras and colleagues emphasize two important concepts. First, although more than half $(62 \%)$ of the patients achieved a TO, this is a relatively low proportion of patients when compared with other individual outcomes more commonly used to define high-quality surgery (e.g. postoperative mortality or complications). This provides a more realistic assessment of the overall perioperative care and expected postoperative

\footnotetext{
(C) Society of Surgical Oncology 2020
}

First Received: 17 April 2020;

Published Online: 8 May 2020

D. A. Anaya, MD, FACS

e-mail: daniel.anaya@moffitt.org course, and highlights there is significant room for improvement when considering $\mathrm{TO}$ as the ideal outcome to strive for. Second, the authors present a novel use of TO for the cancer population by corroborating the association between TO, as a perioperative (short-term) outcome, and long-term oncologic outcomes-overall survival. This is a critical finding as it further validates the relevance of TO in the cancer population, aligning the importance of surgical quality (and outcomes) with the primary goal of a curativeintent cancer operation.

The use of composite measures has been proposed by others and found to be a good quality indicator of surgical care. ${ }^{4}$ Composite measures can reduce the amount of data that must be processed to determine hospital performance overall, and the resultant scores can serve as a benchmark that can be tracked and evaluated on multiple levels. Among the advantages of using TO following liver surgery are the facts that many individual outcomes are interrelated (e.g. post-hepatectomy liver insufficiency and mortality) and that, at the same time, each individual outcome may address different domains of quality (e.g. postoperative mortality is related to surgeon and hospital volume, while failure to rescue is related to structure/processes of perioperative care, and LOS or readmissions may be related to established discharge planning processes). ${ }^{5-7}$ As such, TO used as an all-or-none concept encompasses determinants of quality that include structure, process, and individual outcomes as a unifying measure. ${ }^{2}$ This is particularly relevant for the care of complex cancers, including HCC and ICC, for which appropriate execution and coordination of care across different specialties and through different phases of treatment have proven to be critical in overall outcomes, and are best accomplished through integrated, multidisciplinary, disease-specific (hepatobiliary) programs. ${ }^{8}$ Furthermore, the concept of a unifying quality 
metric has important implications for liver resections in particular. In addition to representing a summary measure of overall care, it can be used as a benchmark to compare performance across different institutions or health systems. ${ }^{9}$ TO have been found to better discriminate hospital performances than other measures typically used to designate hospitals using rankings (e.g. US News and World Report) ${ }^{10}$ or accreditation standards (e.g. Magnet Hospitals). ${ }^{11}$ These findings have potential policy-based and patient-centered implications when considering where treatment for HCC and ICC should be provided. From a patient's perspective, it also represents a more tangible and relevant measure of 'success', and one that can be used when choosing to have care at a given hospital. Furthermore, from this perspective, TO provides a framework to delineate patient expectations.

Based on these considerations, it is easy to see why TO are being utilized more frequently in the healthcare system, as composite measures provide an easily understood summary of performance and represent a good benchmark for quality. However, as we integrate the use of TO measurement into a new standard for surgical quality, it is important to understand the drawbacks or limitations of this approach. ${ }^{12}$ Critical to the use of TO is the individual outcomes included in the composite score. In the current study, LOS was a component of TO driving, in great part, the result, i.e. many patients did not accomplish a TO based on this individual outcome. Although LOS is relevant to patients and has been found to represent some domains of quality of surgical care following liver resection, it is also variable across nations and may represent cultural differences and/or differences in patterns of care, rather than quality of care delivery in the most strict sense. The authors appropriately defined this outcome, differentiating the site of practice (Eastern vs. Western) to account in part for this limitation. Similarly, the method for data collection can vary and the weight that each of the individual outcomes should have can be different. In this case, the development of a robust database was critical to accurate assessment of the outcomes. Furthermore, the approach used by the authors of using an all-or-none method rather than a weighted approach for defining TO is more in line with the clinical course and outcomes, making it easier to grasp from a patient's perspective. It is unclear if, in the future, TO should be built using weighted methodology (i.e. resembling a nomogram), however this approach would distance the benefit of TO from a patient-centered approach. Lastly, it is difficult to use TO or other composite measures to inform performance improvement initiatives because, by design, they lack the granularity of targetable individual variables. It appears clear that for liver resections, as part of the treatment for HCC and ICC, there is significant room for improvement based on an overall TO of $62 \%$; however, one should look into the details of each individual outcome to identify actionable targets.

The findings provided by this study are critical and the described surgical TO is a laudable goal for primary liver tumors following surgery. The authors should be congratulated for this work, framing the use of TO for liver resection, and for the collaborative effort in building a multi-institutional international patient database to improve the accuracy and generalizability of these results. Moving forward, future studies evaluating outcomes following surgery and quality of surgical care should incorporate TO measures. When evaluating care delivery for patients with complex cancers, the role of a multidisciplinary team is critical. This is also the case for primary liver tumors, for which multimodal strategies are now available. ${ }^{13}$ As we integrate TO into the field of cancer surgery, oncologic composite measures/outcomes (i.e. the Textbook Oncologic Outcome [TOO]) should evolve to incorporate variables representing domains of multidisciplinary care delivery.

DISCLOSURE Jason Denbo and Daniel A. Anaya declare no competing interests.

\section{REFERENCES}

1. Tsilimigras DI, Sahara K, Moris D, et al. Assessing textbook outcomes following liver surgery for primary liver cancer over a 12-year time period at major hepatobiliary centers. Ann Surg Oncol. In press.

2. Kolfschoten NE, Kievit J, Gooiker GA, et al. Focusing on desired outcomes of care after colon cancer resections; hospital variations in 'textbook outcome'. Eur J Surg Oncol. 2013;39(2):156-163.

3. Poelemeijer YQM, Marang-van de Mheen PJ, Wouters M, Nienhuijs SW, Liem RSL. Textbook outcome: an ordered composite measure for quality of bariatric surgery. Obes Surg. 2019;29(4):1287-1294.

4. Dimick JB, Staiger DO, Hall BL, Ko CY, Birkmeyer JD. Composite measures for profiling hospitals on surgical morbidity. Ann Surg. 2013;257(1):67-72.

5. Chen Q, Olsen G, Bagante F, et al. Procedure-specific volume and nurse-to-patient ratio: implications for failure to rescue patients following liver surgery. World $J$ Surg. 2019;43(3):910-919.

6. Li M, Zhang W, Jiang L, Yang J, Yan L. Fast track for open hepatectomy: a systemic review and meta-analysis. Int $J$ Surg. 2016;36(Pt A):81-89.

7. Nathan H, Cameron JL, Choti MA, Schulick RD, Pawlik TM. The volume-outcomes effect in hepato-pancreato-biliary surgery: hospital versus surgeon contributions and specificity of the relationship. J Am Coll Surg. 2009;208(4):528-538.

8. Lau K, Salami A, Barden G, et al. The effect of a regional hepatopancreaticobiliary surgical program on clinical volume, quality of cancer care, and outcomes in the Veterans Affairs system. JAMA Surg. 2014;149(11):1153-1161.

9. Merath K, Chen Q, Bagante F, et al. Textbook outcomes among medicare patients undergoing hepatopancreatic surgery. Ann Surg. Epub 29 Nov 2018. https://doi.org/10.1097/sla. 0000000000003105 
10. Mehta R, Tsilimigras DI, Paredes AZ, et al. Comparing textbook outcomes among patients undergoing surgery for cancer at $\mathrm{U}$. S. News \& World Report ranked hospitals. J Surg Oncol. 2020;121(6):927-935.

11. Merath K, Mehta R, Tsilimigras DI, et al. Quality of care among medicare patients undergoing pancreatic surgery: safety grade, magnet recognition, and leapfrog minimum volume standardswhich quality benchmark matters? J Gastrointest Surg. Epub 10 Feb 2020. https://doi.org/10.1007/s11605-019-04504-6.

12. Barclay M, Dixon-Woods M, Lyratzopoulos G. The problem with composite indicators. BMJ Qual Saf. 2019;28(4):338-344.
13. Primrose JN, Fox RP, Palmer DH, et al. Capecitabine compared with observation in resected biliary tract cancer (BILCAP): a randomised, controlled, multicentre, phase 3 study. The Lancet Oncology. 2019;20(5):663-673.

Publisher's Note Springer Nature remains neutral with regard to jurisdictional claims in published maps and institutional affiliations. 